\title{
An Evaluation on Investment of Research Funds with a Neural Network Algorithm in "Double First-Class" Universities
}

\author{
Jihong Sun $\left(\mathbb{D},{ }^{1}\right.$ Yahui Li $\left(\mathbb{D},{ }^{2}\right.$ Xiaoyun Zhao $\mathbb{D}^{\circ},{ }^{2}$ and Nanxing Zhang ${ }^{1}{ }^{1}$ \\ ${ }^{1}$ China National Institute of Education Science, Beijing 100088, China \\ ${ }^{2}$ School of Economics, Qufu Normal University, Qufu 276800, China \\ Correspondence should be addressed to Nanxing Zhang; zhangnx2014@126.com
}

Received 8 May 2020; Accepted 4 August 2020; Published 4 November 2020

Academic Editor: Abdelalim A. Elsadany

Copyright (c) 2020 Jihong Sun et al. This is an open access article distributed under the Creative Commons Attribution License, which permits unrestricted use, distribution, and reproduction in any medium, provided the original work is properly cited.

In the current context of the establishment of world-class universities and disciplines in China, this study examined the investment of research funds at universities. First, six variables were selected as evaluation indicators from the perspective of fixed assets, teaching configuration, research instruments, and the number of books in libraries. Seventy-two universities were investigated from 2013 to 2017. Second, an evaluation system was constructed using the BP (backpropagation) neural network method and its applicability was verified. Finally, by adjusting the six indicators, the investment of university research funds could be adjusted and predicted to provide a reference for the construction of "first-class" universities and disciplines.

\section{Introduction}

Since reform and opening up, China has been attached greater importance to education, particularly at a time when the world is undergoing great development, change, and adjustment, with rapid scientific and technological progress, deepening economic globalization, and fierce competition for high-skilled workers. At the same time, the development of higher education in China has attracted worldwide attention. The initial formation of a socialist system of higher education has been constructed, which is multiform, multilevel, and full of disciplines to meet the needs of economic construction and social development, where a large number of outstanding and top-notch high-skilled workers have been trained for socialist modernisation and play a great role in the country's economic construction, scientific and technological progress, and social development. Today, in the era of the knowledge economy, the previously implemented " 211 " project and " 985 " project of higher education policy have exposed some problems, such as low resource utilisation, identity consolidation, and lack of fair and effective competition among other factors. The country's development requires improving the quality of our people and training innovative high-skilled workers. Considering the current state of China and its critical period of reform as well as the international experience of advanced higher education, the Government has introduced a policy of "double first-class" university system.

The study of investment in scientific research funds from each university in terms of infrastructure and equipment is more favourable to the understanding and grasp of the current "first-class" universities in all aspects. The increase in fixed assets of universities, the purchase of scientific research instruments and equipment, and the expansion of library books are more conducive to determining the scope of funding, facilitating the use of funds in practise, and efficiently promoting the construction and development of first-class universities and first-class disciplines in China with high efficiency.

\section{Literature Review}

2.1. Research on Evaluation of School Facilities. By analysing the current status of the development of teaching laboratories in universities, Gao [1] propose optimising teaching laboratories in terms of both informational construction and social services, so that teaching laboratories can better contribute to the development of "double first-class" 
schools. Zhang [2] discusses the important role of first-class laboratory construction in "double first-class" universities and provides reference opinions for promoting the development of "double first-class" construction projects in three aspects: laboratory management mechanisms, laboratory conditions construction, and laboratory teacher team configuration.

Liu and Ding [3] use the Shanghai University Library as an example to study the availability of subject literature resources in the construction of "double first-class" universities. It is proposed that the establishment of a document resource guarantee system should be suitable for the construction of first-class disciplines in colleges and universities, with a focus on the construction of document resources in discipline groups and on regular evaluation and assessment. Zhang and Zheng [4] summarised the current status of the opening and sharing of large-scale instruments and equipment in colleges and universities. They proposed a new method of open sharing of these resources under the realistic background of the construction of "double first-class" universities. Jiang et al. [5] analysed the importance of the current "double first-class" construction on the new experimental teaching platform. Deep three-dimensional teaching designs, such as online resources and testing, are discussed. The emphasis is on cultivating students' basic experimental skills and innovation awareness as well as enhancing the ability of experimental teachers. Wu and $\mathrm{Ma}$ [6] took 42 "double first-class" university libraries as research objects and highlighted major issues in the opening of scientific research achievements. It is believed that the formulation and improvement of policies should be strengthened to realise early open access to scientific research results. Fan et al. [7] studied and evaluated the requirements of the construction of "double first-class" universities on the construction of library resources. They proposed corresponding measures for the operation of the library's ladder-type resource construction model and related policy mechanisms.

\subsection{Research on the Evaluation of Infrastructure with Neural} Network Universities. Wang [8] used a BP neural network to establish an evaluation index system for the quality of electronic resources of libraries. Through empirical research on the library's electronic resources, the effectiveness of management evaluation is clarified. Liu and $\mathrm{Li}$ [9] used the SOM (self-organizing map) neural network clustering algorithm to study the outstanding problems in the digital construction of university libraries. They screen and process related data resources of users to form related data sets and combine related technologies to establish a personalised recommendation service system for users in university libraries. Zhang et al. [10] conducted a comprehensive and scientific evaluation of the information dissemination capability of the think tank on the We Chat public platform. They conducted simulation verification using the BP neural network model. The evaluation model has strong operability and practicability and can provide new methods and ideas for information dissemination.
Zhang et al. [11] used a BP neural network to establish an evaluation system. They use the capability maturity model to study the think tank service capabilities of university libraries. It is conducive to college libraries to determine their own think tank service capability level and optimise and improve it in a targeted manner.

\subsection{Research on the Evaluation of the Teaching Quality Neural} Network Universities. Zheng and Yan [12] used a teaching evaluation mechanism to measure teaching quality. Through the three aspects of the difference in teaching quality, the unity of teaching evaluation subjects, and the difficult operation of teaching evaluation methods, a comprehensive teaching quality evaluation system was established. Fan and $\mathrm{Ma}$ [13] evaluated the teaching quality of college teachers by establishing a complex nonlinear relationship between the evaluation results of teaching quality and various indicators. Zhu and Wang [14] used a particle swarm optimisation method to establish a BP neural network evaluation model. It solved the problem of scientific research performance evaluation faced by the Scientific Research Management Department of Colleges and Universities. It used particle swarm optimisation to optimise the initial weights and thresholds of the BP neural network model and then made predictions.

In summary, most of the research on the construction of "double first-class" universities has been carried out in combination with the basic supporting facilities of universities, the quality of education and teaching, and other aspects. There are few studies on research funding at universities, and there is a lack of research that uses neural networks for in-depth analysis. This study uses a neural network model to discuss the investment in scientific research funds of various universities from the aspects of infrastructure and hardware equipment of "double first-class" universities and discusses the related implications.

\section{BP Neural Network Model}

An artificial neurone emulates the abstraction, simplification, and simulation of biological neurones. It is the basic processing unit of an artificial neural network. Neural network learning is one of the basic algorithms in the field of artificial intelligence. It was a mathematical model proposed by the psychologist McCulloch and mathematician Pitts in 1943. Its main application areas involve pattern recognition, intelligent robots, nonlinear system recognition, knowledge processing, and other factors.

\subsection{Mathematical Model of Neural Network Perceptron.} The most common neural network model with only a single hidden layer is a three-layer perceptron. These three layers are the input layer, hidden layer, and output layer. There is a mathematical relationship between the signals of each layer as follows:

For the hidden layer, 


$$
\begin{cases}y_{i}=f\left(\text { net }_{j}\right), & j=1,2, \ldots, m \\ \text { net }_{j}=\sum_{i=0}^{n} v_{i j} x_{i}, & j=1,2, \ldots, m\end{cases}
$$

For the output layer,

$$
\begin{cases}o_{k}=f\left(\text { net }_{k}\right), & k=1,2, \ldots, l, \\ \text { net }_{k}=\sum_{j=0}^{m} w_{j k} y_{j}, & k=1,2, \ldots, l .\end{cases}
$$

In formulas (1) and (2), the transformation function $f(x)$ is usually a unipolar sigmoid function, which we call

$$
f(x)=\frac{1}{1+e^{-x}} \text {. }
$$

The sigmoid function is derivative and continuous. For equation (3), we call

$$
f^{\prime}(x)=f(x)[1-f(x)] .
$$

If necessary, you can also use the bipolar sigmoid function:

$$
f(x)=\frac{1-e^{-x}}{1+e^{-x}}
$$

To reduce the computational complexity, the output layer can also use linear functions as needed:

$$
f(x)=k x .
$$

3.2. Derivation of the BP Algorithm. The following uses the three-layer BP neural network model as an example to derive the BP learning algorithm.

(1) Network error

If the network output is not equal to the expected output, then there is an output error $E$ as follows:

$$
E=\frac{1}{2}(d-O)^{2}=\frac{1}{2} \sum_{k=1}^{l}\left(d_{k}-O_{k}\right)^{2}
$$

Expanding the above error to the hidden layer,

$E=\frac{1}{2} \sum_{k=1}^{l}\left[d_{k}-f\left(\text { net }_{k}\right)\right]^{2}=\frac{1}{2} \sum_{k=1}^{l}\left[d_{k}-f\left(\sum_{j=0}^{m} w_{j k} y_{j}\right)\right]^{2}$.

Further, expand to the input layer:

$$
E=\frac{1}{2} \sum_{k=1}^{l}\left\{d_{k}-f\left[\sum_{j=0}^{m} w_{j k} f\left(\text { net }_{j}\right)\right]\right\}^{2}=\frac{1}{2} \sum_{k=1}^{l}\left\{d_{k}-f\left[\sum_{j=0}^{m} w_{j k} f\left(\sum_{i=0}^{n} v_{i j} x_{i}\right)\right]\right\}^{2} .
$$

(2) Network weight adjustment based on gradient descent

From equation (9), the input error of the neural network is a function of weights $w_{j k}$ and $v_{i j}$ of each layer. By adjusting the weight value, the error $E$ can be changed, so that the adjustment amount of the weight value is proportional to the gradient decrease of the error, namely,

$$
\begin{gathered}
\Delta w_{j k}=-\eta \frac{\partial E}{\partial w_{j k}}, \quad k=1,2, \ldots, l, j=1,2, \ldots m, \\
\Delta v_{i j}=-\eta \frac{\partial E}{\partial v_{i j}}, \quad i=1,2, \ldots, n j=1,2, \ldots, m .
\end{gathered}
$$

In the above formula, the minus sign indicates gradient descent. The constant $\eta \in(0,1)$ is a scale factor, which reflects the learning rate. Surely, the BP algorithm belongs to the $\sigma$ learning rule.

Equations (10) and (11) only express the idea of adjusting the weights mathematically. The calculation formula derivation of the weight adjustment of the three-layer BP algorithm is as follows. It is assumed that in all the derivation processes, there is $j=0,1,2, \ldots, m$, $k=1,2, \ldots, l$ for each output layer and $i=0,1,2, \ldots, n, j=1,2, \ldots, m$ for each hidden layer.

For the output layer, equation (10) can be written as

$$
\Delta w_{j k}=-\eta \frac{\partial E}{\partial w_{j k}}=-\eta \frac{\partial E}{\partial \text { net }_{k}} \frac{\partial \text { net }_{k}}{\partial w_{j k}} .
$$

Equation (11) can be written as

$$
\Delta v_{i j}=-\eta \frac{\partial E}{\partial v_{i j}}=-\eta \frac{\partial E}{\partial \text { net }_{j}} \frac{\partial \text { net }_{j}}{\partial v_{i j}} .
$$

We define an error signal for the output layer and the hidden layer.

$$
\begin{gathered}
\delta_{k}^{o}=-\frac{\partial E}{\partial \text { net }_{k}}, \\
\delta_{j}^{y}=-\frac{\partial E}{\partial \text { net }_{j}} .
\end{gathered}
$$

Comprehensive application of formulas (2) and (14): the weight adjustment formula of (12) can be rewritten as 


$$
\Delta w_{j k}=\eta \delta_{k}^{o} y_{j}
$$

Comprehensive application of formulas (4) and (15): the weight adjustment formula of (13) can be rewritten as

$$
\Delta v_{i j}=\eta \delta_{j}^{y} x_{i}
$$

Calculate the error signals $\delta_{k}^{o}$ and $\delta_{j}^{y}$ in formulas (14) and (15); then, the calculation and derivation of the weight adjustment amount are also completed.

The output layer $\delta_{k}^{o}$ can be expanded to

$$
\delta_{k}^{o}=-\frac{\partial E}{\partial \text { net }_{k}}=-\frac{\partial E}{\partial o_{k}} \frac{\partial o_{k}}{\partial \text { net }_{k}}=-\frac{\partial E}{\partial o_{k}} f^{\prime}\left(\text { net }_{k}\right) .
$$

The hidden layer $\delta_{j}^{y}$ can be expanded to

$$
\delta_{j}^{y}=-\frac{\partial E}{\partial \text { net }_{j}}=-\frac{\partial E}{\partial y_{j}} \frac{\partial y_{i}}{\partial \text { net }_{j}}=-\frac{\partial E}{\partial y_{i}} f^{\prime}\left(\text { net }_{j}\right) .
$$

Next, according to formulas (18) and (19), we find the partial derivative of the network error to the output of each layer.

Output layer: using equation (7), the partial derivative can be obtained:

$$
\frac{\partial E}{\partial o_{k}}=-\left(d_{k}-o_{k}\right)
$$

Hidden layer: using equation (8), the partial derivative can be obtained:

$$
\frac{\partial E}{\partial y_{j}}=-\sum_{k=1}^{l}\left(d_{k}-o_{k}\right) f^{\prime}\left(\operatorname{net}_{k}\right) w_{j k} .
$$

Substituting the above results into formulas (18) and (19), and applying equation (4), $f^{\prime}(x)=f(x)$ $[1-f(x)]$, we obtain

$$
\begin{gathered}
\delta_{k}^{o}=\left(d_{k}-o_{k}\right) o_{k}\left(1-o_{k}\right), \\
\delta_{j}^{y}=\left[\sum_{k=1}^{l}\left(d_{k}-o_{k}\right) f^{\prime}\left(\text { net }_{k}\right) w_{j k}\right] f^{\prime}\left(\text { net }_{j}\right) \\
=\left(\sum_{k=1}^{l} \delta_{k}^{o} w_{j k}\right) y_{j}\left(1-y_{j}\right) .
\end{gathered}
$$

Substituting equations (22) and (23) into equations (16) and (17), the calculation formula for the weight adjustment of the BP learning algorithm of the three-layer perceptron can be obtained:

$$
\begin{aligned}
\Delta w_{j k} & =\eta \delta_{k}^{o} y_{j}=\eta\left(d_{k}-o_{k}\right) o_{k}\left(1-o_{k}\right) y_{j}, \\
\Delta v_{i j} & =\eta \delta_{j}^{k} x_{i}=\eta\left(\sum_{k=1}^{l} \delta_{k}^{o} w_{j k}\right) y_{j}\left(1-y_{j}\right) x_{i} .
\end{aligned}
$$

(3) Vector form of BP learning algorithm

Output layer: assuming $Y=\left(y_{1}, y_{2}, \ldots, y_{j}\right.$, $\left.\ldots, y_{m}\right)^{T}, \delta^{o}=\left(\delta_{1}^{o}, \delta_{2}^{o}, \ldots, \delta_{k}^{o}, \ldots, \delta_{l}^{o}\right)^{T}$, the weight matrix adjustment from the hidden layer to the output layer is

$$
\Delta W=\eta\left(\delta^{o} Y^{T}\right)^{T} .
$$

Hidden layer: assuming $X=\left(x_{1}, x_{2}, \ldots, x_{i}, \ldots, x_{m}\right)^{T}$, $\delta^{y}=\left(\delta_{1}^{y}, \delta_{2}^{y}, \ldots, \delta_{k}^{y}, \ldots, \delta_{l}^{y}\right)^{T}$, the weight matrix adjustment from the input layer to the hidden layer is

$$
\Delta V=\eta\left(\delta^{y} X^{T}\right)^{T}
$$

According to formulas (25) and (26), in the BP learning algorithm, the adjustment formula of the weights of each layer is identical in the same form. They all require three factors, the learning rate $\eta$, the error signal $\delta$ output by this layer, and the input signal $Y$ (or $X$ ) of this layer. The error signal of each hidden layer is related to the error signal of each previous layer, and it is transmitted from the output layer and back to the input layer.

\subsection{Determination of Input Variables and Output Variables}

3.3.1. Input Dimensionality Reduction. For image data, adjacent pixels are highly correlated, so the input data is somewhat redundant. Suppose a $16 \times 16$ grey value image is processed. The input is a 256-dimensional vector $x \in R^{256}$, where the feature value $x_{j}$ corresponds to the brightness value of each pixel. Because of the correlation between adjacent pixels, the input vector needs to be converted into an approximate vector with a lower dimension. At this time, the error is very small, which does not affect the processing result, but the amount of calculation is greatly reduced.

Principal Component Analysis (PCA) is a statistical analysis method to identify the main contradictions of things. The purpose of calculating the principal component is to find $r(r<n)$ new variables. Each new variable is a linear combination of the original $n$ variables. They reflect the influence of the original $n$ variables, and these new variables are uncorrelated.

3.3.2. Preprocessing of Input and Output Data. Scale normalisation is a linear transformation. It readjusts the value of each input component of the data. These dimensions may be independent of each other. Ensure that the final data vector falls within the interval $[0,1]$ or $[-1,1]$. The pixel values obtained when processing natural images are in the range $[0$, 255]. A common process is to divide these pixel values by 255 to scale them to $[0,1]$. Transform the input and output data to the value of the interval $[0,1]$ :

$$
x_{i}^{\prime}=\frac{x_{i}-x_{\min }}{x_{\max }-x_{\min }},
$$


where $x_{i}$ represents the input or output data, $x_{\min }$ represents the minimum value of the data change, and $x_{\max }$ represents the maximum value of the data change range.

If the input or output data is converted to a value in the range $[-1,1]$, the following conversion formulas are commonly used:

$$
\begin{aligned}
x_{\text {mid }} & =\frac{x_{\max }+x_{\min }}{2}, \\
x_{i}^{\prime} & =\frac{x_{i}-x_{\operatorname{mid}}}{1 / 2\left(x_{\max }-x_{\min }\right)} .
\end{aligned}
$$

$x_{\text {mid }}$ represents the middle value of the data change range. After changing according to the above transformation, the intermediate value of the original data is converted to zero. The maximum value is converted to 1 , and the minimum value is converted to -1 .

3.3.3. Training Sample Set. The laws in neural network training can be extracted from the samples. Therefore, a high-quality data sample set is an important and critical step. The selection of samples should not only be representative, but also eliminate invalid data and erroneous data. At the same time, we must unify the sample category and sample size. Normally, if the number of training samples is large, the training results will more accurately reflect their internal laws. However, when the number of samples reaches a certain upper limit, it is more difficult to improve the accuracy of the network. Generally, the number of training samples is $5-10$ times the total number of network connection weights.

3.4. BP Network Structure Design. Through training samples, the number of nodes in the input and output layers of the network can be determined. The structural design of the BP network is mainly used to determine the number of hidden layers, the number of nodes in each hidden layer, and the neurone activation function of each node.

Determination of the number of hidden layers: according to theory, if a feedforward network has a single hidden layer when designing a multilayer BP network, it will first consider designing only one hidden layer. When there are too many hidden nodes in a hidden layer and the network performance cannot be improved, the second hidden layer will be considered.

Determining the number of hidden layer nodes: the role of hidden layer nodes is to extract internal laws from samples and store them. The commonly used empirical formulas for determining the number of hidden nodes are as follows:

$$
\begin{aligned}
& m=\sqrt{n+l}+\alpha, \\
& m=\mathrm{lbn}, \\
& m=\sqrt{\mathrm{nl}} .
\end{aligned}
$$

In the above formula, $m$ is the number of hidden layer nodes and $n$ is the number of input layer nodes. $l$ is the number of nodes in the output layer, and $\alpha$ is a constant between 1 and 10 .
Approximation and generalisation considerations: the total number of weights and thresholds reflects the information capacity of the network. The "overdesign" of the number of hidden nodes may lead to "overfitting." The noise in the sample is retained, but it reduces the generalisation ability. The above design uses a combination of experience and trial. The theoretical guidance of neural network design still needs to be improved. The research shows that the number of training samples $P$, the given training error $\varepsilon$, and the network information capacity $n_{w}$ should meet the following matching relationship:

$$
P=\frac{n_{w}}{\varepsilon} .
$$

\section{An Empirical Analysis of the Scientific Research Fund with BP Neural Network in Higher Education}

In this study, six evaluation indicators-fixed assets, number of teaching and research instruments and equipment, amount of teaching and research instruments and equipment, number of large teaching and research equipment over 400,000, amount of large teaching and research equipment over 400,000, and number of books in libraries-are selected as input variables. Fixed assets, teaching and research equipment, and library collection are the tools used by students and teachers to acquire new knowledge and solve research problems, which are important in improving the quality of education and research. In addition, with the continuous improvement of the level of science and technology and the continuous acceleration of the process of informationisation in this era, teaching and research instruments and equipment are becoming much more advanced, playing an increasingly important role in conducting relevant teaching experiments. In 2018, China's dual-class universities invested about 388.2 billion yuan in fixed assets, a total of 6.31 million pieces of teaching and research instruments, with an investment of about 126.6 billion yuan, of which more than 400,000 large teaching and research equipment of about 32.6 billion yuan, or $25.8 \%$ of the total investment in teaching and research equipment. The library collection was about 250 million books. Generally speaking, fixed assets, research instruments, and library collections comprise the infrastructure for teaching and research in universities, and they are also the basic requirements for the construction of China's "double firstclass" universities.

As study subjects, 72 colleges and universities among the "double first-class" colleges and universities in 2017 were selected, and the aforementioned indicators were used as network input, the number of scientific research activities was used as the network output, and the number of implicit layer nodes was determined according to the empirical formula of the number of implicit nodes, thus forming a 6-31 neural network structure. The BP neural network is created using the software $R$, and weights and thresholds are determined using correlation functions. The minimummaximum normalisation method is used to normalise the 


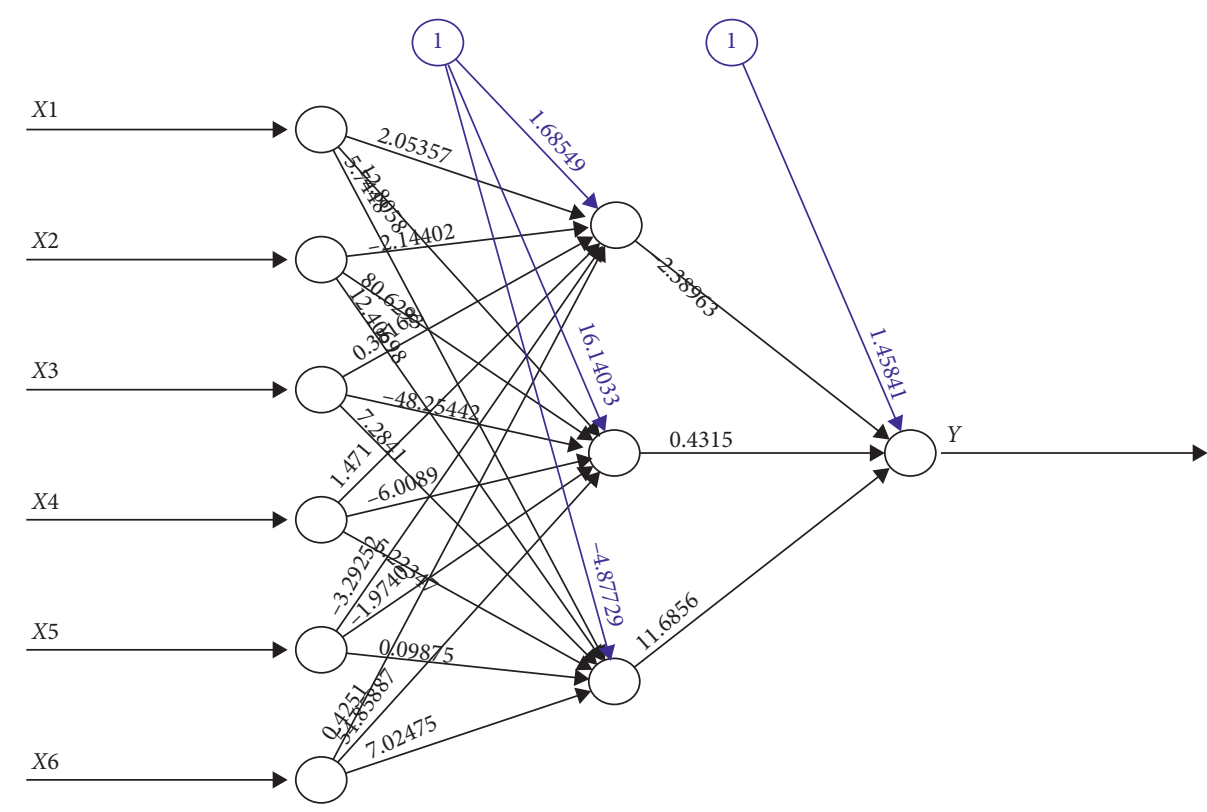

FiguRE 1: The neural network model of university samples in 2017.

sample indicator data with the total score, and the neural net function is used to create the forward network to achieve an arbitrary mapping of the neural network from input to output. The algorithm for computing the neural network is rprop+, the incentive function from the input layer to the implicit layer is Tansig, the incentive function from the implicit layer to the output layer is Purelin, the learning rate is set to 0.01 , and the number of neurones in the implicit layer is 3 . The stop queue for the error function is 0.001 , the maximum number of iterative training allowed is 100000 , and the number of training sessions is 1000 . Set the error performance index of network convergence as MSE (mean square error); the set output mode is linear output, and the default value is selected for all other parameters. By default, the algorithm used in the neural net function is based on elastic backpropagation with upweighted regression and an additional modification of either the learning rate associated with the minimum absolute gradient or the minimum learning rate itself.

The colleges in the sample in 2017 are divided into training and test sets, and the constructed neural network is shown in Figure 1. $X 1$ is a fixed asset. $X 2$ is the number of teaching and research instruments and equipment. $X 3$ is the amount of teaching and research equipment. $X 4$ is the number of large-scale teaching and research equipment. $X 5$ is the amount of large-scale teaching and research equipment. $X 6$ is the number of books in libraries. $Y$ is the funding for scientific research activities.

The maximum step of training the neural network was 88143. When this value is reached, the training process of the neural network stops. Specify the threshold value of the partial derivative of the error function, which is the stop threshold of the error function, to reach 0.00098 . The error rate of this neural network model was 0.07809 . The black line (the line starting from the input node) shows the connection between each layer and the weight on each connection, while the blue line (the lines start from the deviation nodes distinguished by 1) shows the deviation added at each step. It can be considered that the deviation is the intercept of the linear model. The deviation of the first node of the hidden layer was 1.68549. The deviation of the second node of the hidden layer was 16.14033 . The deviation of the third node of the hidden layer was -4.87729 . The deviation of the output layer node was 1.45841 . The specific values of the weights between the nodes of each layer are shown in Table 1.

From Table 1, the weights of the first node of the hidden layer have a greater impact on the indicator of the number of teaching and research equipment and the indicator of the number of large teaching and research equipment, both of which play an inhibitory role. The weights for the second node of the hidden layer have a greater impact on the number of teaching and research equipment, which is a facilitator, and the number of books in libraries, which is disincentive. The weights of the third node of the hidden layer have a greater impact on the number of teaching and research equipment indicators and the number of teaching and research equipment indicators, with the former acting as an inhibitor and the latter as a facilitator. The relationship between the hidden layer and the output layer has a greater impact on the third and first nodes.

The MSE of the neural network with a value of 651133098 is significantly smaller than that of the linear regression model with a value of 1723270943 , which indicates that the neural network model is better. Furthermore, it is possible to predict the funding for higher education research activities of "double-first-class" universities through a neural network evaluation model. As long as the data of those indicators are collected for a certain university in a certain year, it is possible to predict the approximate range of funding for scientific research activities of that university, which provides an effective reference for the funding of college education in China. 
TABLE 1: Weight table of neural network of university samples in 2017.

\begin{tabular}{|c|c|c|c|c|}
\hline & Hidden layer first node & $\begin{array}{l}\text { Hidden layer } \\
\text { second node }\end{array}$ & $\begin{array}{c}\text { Hidden layer } \\
\text { third node }\end{array}$ & Output layer \\
\hline Fixed assets & 2.053567 & -12.80568 & 5.744475 & - \\
\hline Number of teaching and research equipment & -2.144015 & 80.63330 & -12.48698 & - \\
\hline Amount of teaching and research equipment & 0.3614817 & -48.25442 & 7.784177 & - \\
\hline Number of large-scale teaching and research equipment & 1.471630 & -6.008896 & -5.223469 & - \\
\hline Amount of large-scale teaching and research equipment & -3.292561 & -1.974632 & 0.098747 & - \\
\hline Number of books in libraries & 0.425100 & -54.85887 & 7.024750 & - \\
\hline Hidden layer first node & - & - & - & -2.389628 \\
\hline Hidden layer second node & - & - & - & 0.4314989 \\
\hline Hidden layer third node & - & - & - & 11.68560 \\
\hline
\end{tabular}

\section{Summary}

In the current context of the creation of world-class universities and disciplines in China, this paper examines the investment of research funds at universities. Six variables were selected as evaluation indicators from the perspective of fixed assets, teaching configuration and research equipment, and the number of books in libraries, and the data of 72 universities from 2013 to 2017 were analysed. The MSE is 651133098 for the neural network and 1723270943 for the regression model, which proved that the neural network model is more effective than the regression model. The theoretical model can be applied to practice, and the findings show that it is possible to modify and predict the investment strategy of the scientific research expenses of universities through adjusting the six indicators, which provides an effective reference for the construction of "world-class" universities and disciplines.

\section{Appendix}

\section{A. Neural Network Algorithm}

library ("neuralnet")

\#Load package

set.seed (1)

max_data <-apply (data, 2, max)

min_data $<$-apply (data, 2, min)

data_scaled $<$-scale $\quad$ (data, $\quad$ center $=$ min_data, scale = max_data-min_data)

\#The function scale is a general function for data standardization, The default method is to center or scale the columns of a numeric matrix.

index $=$ sample $\left(1:\right.$ nrow $\quad$ (data), round $\left(0.70^{*}\right.$ nrow (data)))

$\# 70 \%$ of the data is used to train the neural network, and the remaining $30 \%$ is used to test the neural network.

train_data <-as.data.frame (data_scaled [index,])

test_data $<$-as.data.frame (data_scaled [-index,])
\#The data is divided into two new data frames, called train_data and test_data

$n=$ names (data)

$f=$ as.formula $\quad$ (paste $\quad$ " $Y \sim$ ”,paste $\quad(n[! n \%$ in\%

“Y”],collapse = “+”)))

\#Build the formula that will be used to build the neural network

net_data $=$ neuralnet $\quad(f, \quad$ data $=$ train_data, hidden $=3$, threshold $=0.001$, learningrate $=$

0.01 , linear.output $=T$ )

\#Use neuralnet functions to build and train neural networks. There are 3 hidden layers, the stop threshold of the error function is 0.001 , and the learning rate is 0.01 .

net_data\$result.matrix

\#Output weight value and threshold value

plot (net_data)

\#Drawing a neural network

predict_net_test $<$-compute (net_data, test_data $[, 1: 6]$ )

predict_net_test_start $<$-predict_net_test\$ne-

t.result ${ }^{*}(\max (\operatorname{data} \$ Y) \pm \min (\operatorname{data} \$ Y))+\min (\operatorname{data} \$ Y)$

\#Using neural networks to make predictions.

test_start < -as.data.frame $\quad\left((\text { test_data } \$ Y)^{*}(\max \right.$

$(\operatorname{data} \$ Y) \pm \min \quad(\operatorname{data} \$ Y))+\min \quad(\operatorname{data} \$ Y))$

MSE.net_data <-sum ((test_start-pre-

dict_net_test_start)2)/nrow (test_start) \#Define the mean square error formula.

Regression_Model $<-\operatorname{lm}(Y \sim$,data $=$ data $)$

\#Establish a linear regression model to understand the accuracy of neural network predictions.

summary (Regression_Model)

test $<$-data [-index,

predict_lm $<$-predict (Regression_Model, test)

MSE.lm <-sum ((predict_lm-test\$Y)2)/nrow (test)

MSE.net_data

\#Calculate the MSE value of the neural network

MSE. $\operatorname{lm}$

\#Calculate the MSE value of the regression model 


\section{Data Availability}

The data used to support the findings of this study are included within the article.

\section{Conflicts of Interest}

The authors declare that they have no conflicts of interest.

\section{Acknowledgments}

This article is a phased result of the research program "Decade Research on the Performance Evaluation of Colleges and Universities," National Institute of Education Sciences (Grant no. GYH2019010).

\section{References}

[1] L. Gao, "Teaching laboratory optimization construction promotes the construction of "double first-class" in colleges and universities," New Campus, no. 7, pp. 47-48, 2017.

[2] H. Zhang, "Research on construction of first-class laboratories under "Double world-class" background," Experimental Technology and Management, vol. 34, no. 12, pp. 6-10, 2017.

[3] H. Liu and D. Ding, "The strategy of document resources guarantee in local university libraries under the background of "double First-class" - - taking Shanghai University Library as an example," Journal of Library Science, no. 3, pp. 9-14, 2019.

[4] H. Zhang and X. Zheng, "New measures for opening and sharing of university large-scale instruments and equipment under background of "Double first-class"” Experimental Technology and Management, vol. 36, no. 6, pp. 8-11, 2019.

[5] W. Jiang, Y. Zhang, and Y. Su, "Research on establishment and application of experimental teaching platform in "Double first-class" construction," Experimental Technology and Management, vol. 36, no. 6, pp. 16-20, 2019.

[6] Q. Wu and L. Ma, "An empirical study on the open access of scientific research achievements of domestic "double first class" university libraries," Research on Library Science, no. 12, pp. 72-81, 2019.

[7] C. Fan, J. Yu, and D. Li, "A study on ladder-type resources construction model of academic libraries under the background of "double first-class," Construction Library Work in Colleges and Universities, no. 4, pp. 62-66, 2019.

[8] J. Wang, "Study on the construction of electronic resources' quality evaluation system in university libraries: based on the BP neural network model," New Century Library, no. 3, pp. 30-33, 2017.

[9] A. Liu and Y. Li, "The recommendation system of university library personalized service based on SOM," Library Tribune, no. 4, pp. 95-102, 2018.

[10] L. Zhang, X. Zhang, Z. Li, and H. Lu, "Evaluation of think tank's information dissemination capacity on wechat public platform based on BP neural network," Information Studies: Theory \& Application, vol. 41, no. 10, pp. 93-99, 2018.

[11] X. Zhang, B. Zhao, H. Lu, and Y. Li, "Research on the maturity model and evaluation of university library think-tank service capability," Library, no. 7, pp. 26-33, 2019.

[12] Y. Zheng and C. Yan, "Research on evaluation model of university teachers' teaching quality based on BP neural network," Journal of Chongqing University of Technology (Natural Science), no. 1, pp. 85-90, 2015.
[13] Y. Fan and L. Ma, "Teaching quality evaluation model of colleges and universities optimized BP neural network," Statistics \& Decision, no. 2, pp. 80-82, 2018.

[14] Q. Zhu and J. Wang, "Research on university scientific research management evaluation based on particle swarm optimization algorithm and BP neural network," Modern Electronics Technique, vol. 42, no. 7, pp. 87-89+94, 2019. 